\title{
Gold nanoparticles attenuate metastasis by tumor vasculature normalization and epithelial-mesenchymal transition inhibition
}

This article was published in the following Dove Press journal:

International Journal of Nanomedicine

4 May 2017

Number of times this article has been viewed

\author{
Wei $\mathrm{Li}^{\prime}$ \\ Xin $\mathrm{Li}^{\prime}$ \\ Shuhao Liu' \\ Wende Yang' \\ Fan Pan' \\ Xiao-Yan Yang ${ }^{1,2}$ \\ Bin $\mathrm{Du}^{3}$ \\ Li Qin ${ }^{4}$ \\ Yunlong Pan' \\ 'Department of General Surgery, \\ The First Affiliated Hospital of \\ Jinan University, ${ }^{2}$ Key Laboratory \\ of Functional Protein Research \\ of Guangdong Higher Education \\ Institutes, Institute of Life and Health \\ Engineering, College of Life Science \\ and Technology, Jinan University, \\ ${ }^{3}$ Department of Pathology, The First \\ Affiliated Hospital of Jinan University, \\ ${ }^{4}$ Department of Histology, and \\ Embryology, Medical School of Jinan \\ University, Guangzhou, People's \\ Republic of China
}

\begin{abstract}
Angiogenesis is a process by which vessels are formed through preexisting ones, and this plays a key role in the progression of solid tumors. However, tumor vessels are influenced by excessive pro-angiogenic factors, resulting in deformed structures that facilitate the intravasation of tumor cells into the circulation and subsequent metastasis. Moreover, abnormal tumor vessels have low blood perfusion and thereby decreased oxygen infusion into tumors. This results in a hostile microenvironment that promotes epithelial-mesenchymal transition (EMT), a process in which epithelial cells lose their polarity and gain increased motility, which is associated with metastasis and invasion. Here, we demonstrate that gold nanoparticles (AuNPs) facilitate tumor vasculature normalization, increase blood perfusion and alleviate hypoxia in melanoma tumors. Additionally, AuNPs were observed to reverse EMT in tumors, accompanied by the alleviation of lung metastasis. These AuNPs inhibited the migration of B16F10 cells and reversed EMT in B16F10 cells, indicating that AuNPs could directly regulate EMT independent of improvements in hypoxia. Taken together, our data demonstrated that AuNPs could induce tumor vasculature normalization and reverse EMT, resulting in decreased melanoma tumor metastasis.
\end{abstract}

Keywords: gold nanoparticles, tumor vasculature normalization, tumor metastasis, epithelialmesenchymal transition

\section{Introduction}

Angiogenesis is recognized as a key event in the progression of solid tumors. This is a complex process regulated by a balance between pro-angiogenic and anti-angiogenic factors. ${ }^{1}$ However, angiogenesis in solid tumors is always pathological and is characterized by immature vessels with irregular structure and function. ${ }^{2}$ Affected vessels typically create hypoxic and acidic microenvironments in tumors that worsen malignancy. Moreover, the resultant hostile milieu promotes invasion and metastases through an epithelial-mesenchymal transition (EMT). ${ }^{3}$

The prevention or reduction of angiogenesis is one of the most utilized methods in tumor treatment. However, a few clinical studies have demonstrated that such a monotherapy fails to provoke significant response rates or improve overall survival (OS), even in colon cancer (average 6-8 weeks) due to resistance. ${ }^{4,5}$ A randomized trial of anti-VEGF plus irinotecan and 5-fluorouracil showed no difference either in median OS ( 22.0 vs 25.0 months, $P=0.1391$ ) or in the response rate ( $36.8 \%$ vs $35.2 \%$ ) in patients with advanced colorectal cancer. ${ }^{6}$ Similarly, there was no difference in the median OS: 25.4 (95\% CI: 22.2-28.9) months in the anti-VEGF continuation arm versus 23.8 (95\% CI: 21-26.8) months in the no continuation arm (hazard ratio [HR] 0.83; 95\% CI:
Correspondence: Yunlong Pan Department of General Surgery, The First Affiliated Hospital of Jinan University, 613 Huangpu West Road, Guangzhou, Guangdong 510630, People's Republic of China

Tel +862038688429

Email tpanyl@jnu.edu.cn 
$0.63-1.1 ; P=0.19) .{ }^{7}$ Furthermore, anti-VEGF does not prolong disease-free survival (DFS) when added to adjuvant chemotherapy in patients with stage III colon cancer, and the DFS HR for anti-VEGF combined with folinic acid/fluorouracil/ Oxaliplatin (FOLFOX4) versus FOLFOX4 was 1.17 (95\% CI: $0.98-1.39 ; P=0.07) .{ }^{8}$ A previous study has shown that anti-angiogenic therapy may have the unintended effect of increasing tumor invasiveness and distant metastasis. ${ }^{9}$ New therapies involving the normalization of tumor blood vessels have been proposed to address conflicting traditional anti-angiogenic therapy and chemotherapy. ${ }^{10}$

Gold nanoparticles (AuNPs) are customarily used as versatile vehicles for actively or passively delivering biomolecules to target organs. Drug-loaded nanoparticles could be used to target tumors through the enhanced permeability and retention (EPR) effect, wherein nanoparticles of certain sizes tend to accumulate in tumors with highly permeable blood vessels. ${ }^{11,12}$ Recently, we demonstrated that AuNPs could conjunct with recombinant human endostatin, elevate tumor-selective delivery, induce transient tumor vasculature normalization and, thereby, improve tumor vessel perfusion and oxygen supply for improved treatment outcomes. ${ }^{13}$ Our previous studies showed that monotherapy by AuNPs reduced angiogenesis in liver tumors ${ }^{14}$ and inhibited VEGF165induced human umbilical vein endothelial cell (HUVEC) migration and tube formation via the Akt pathway. ${ }^{15,16}$

In this study, we aimed to investigate the mechanism of AuNPs in reducing melanoma lung metastasis. Immunofluorescence and immunohistochemistry were carried out to evaluate the vascular morphology, perfusion, permeability and hypoxia in tumors. The lungs were dissected for metastasis evaluation. For in vitro study, cell counting kit-8 (CCK-8) assay and wound healing assay were used to explore the proliferation and migration of cells. The protein expressions of matrix metalloproteinase-2 (MMP-2), c-Myc, zonula occludens-1 (ZO-1), vimentin, and E-cadherin were analyzed by Western blot. Altogether, our study may provide an experimental basis for the clinical application of AuNPs in the future.

\section{Materials and methods Cell lines}

B16F10 cell lines were purchased from the Type Culture Collection of the Chinese Academy of Sciences (Shanghai, China). Cells were cultured in Dulbecco's Modified Eagle's Medium (DMEM) supplemented with 10\% fetal bovine serum (FBS; Thermo Fisher Scientific, Waltham, MA, USA). HUVECs were obtained from the American Type Culture Collection (ATCC ${ }^{\circledR}$ CRL-1730 ${ }^{\mathrm{TM}}$; Manassas, VA, USA) and cultured in endothelial cell medium (ECM; ScienCell, San
Diego, CA, USA) supplemented with 5\% FBS, $1 \%$ endothelial cell growth supplement (ECGS) and 1\% penicillin/streptomycin. Cells were used between passages 3-7. All cells were maintained in a $37^{\circ} \mathrm{C}$ incubator with a $5 \% \mathrm{CO}_{2}$ environment.

\section{Preparation and characterization of AuNPs}

The AuNPs were prepared according to previously described methods. ${ }^{11}$ Briefly, $0.01 \mathrm{~mol} / \mathrm{L}$ of $\mathrm{HAuCl}_{4}(5 \mathrm{~mL})$ was added to $50 \mathrm{~mL}$ Milli- ${ }^{\circledR}$ water, and the resulting solution was heated and stirred to boiling, and then $10 \mathrm{~mL}$ of $1 \%$ sodium citrate was introduced into the system. The solution was continuously heated and stirred until the development of a wine red color. Subsequently, the solution was filtered through a $0.22 \mu \mathrm{m}$ filter and stored at $4^{\circ} \mathrm{C}$ protected from light. The collected AuNPs were characterized using ultravioletvisible (UV-vis) on a model V-570 Jasco dual-beam spectrophotometer. Particle morphology was visualized using field emission transmission electron microscopy (FETEM; JEM2100; JEOL, Tokyo, Japan). The particle size and zeta potential were measured by dynamic light scattering (DLS) using a Malvern Zetasizer Nano ZS (Malvern Instruments Ltd, Malvern, UK).

\section{Tumor model and therapeutic treatments}

Female C57BL6 mice, 4-6 weeks of age, with body weights of 18-25 g, were purchased from the Guangdong Medical Lab Animal Centre (Guangzhou, China) and housed in a specific pathogen-free environment. All animal experiments and research procedures were approved by and conducted in accordance with the guidelines of the Laboratory Animal Ethics Committee of Jinan University (Permit Number: 20150909160150).

Tumors were established by injecting B16F10 cells $\left(3 \times 10^{6} / 50 \mu \mathrm{L}\right)$ in the right footpad. Tumor volumes were measured with calipers and calculated as length $\times$ width $^{2} \times 0.523$ every day. When the tumor size reached between 100 and $125 \mathrm{~mm}^{3}$, mice were daily injected intravenously with AuNPs $(1 \mathrm{mg} / \mathrm{kg})$ or with an equivalent volume of normal saline (NS) for 4 days. Subsequently, intermittent injections were provided for an additional 10 days.

\section{Tumor vascular perfusion and permeability}

Tumor vascular perfusion was detected by intravenous injections of $10 \mathrm{mg} / \mathrm{kg}$ fluorescein isothiocyanate (FITC)conjugated lectin (Sigma-Aldrich, St Louis, MO, USA) ( $\mathrm{n}=7 /$ group). After $20 \mathrm{~min}$, tumors were excised and immediately frozen in liquid nitrogen, then embedded in 
optimal cutting temperature (OCT) compound (Sakura Finetek, Torrance, CA, USA) and stored at $-80^{\circ} \mathrm{C}$. A series of $20 \mu \mathrm{m}$ cryosections were cut and incubated with antiCD31 antibody (Abcam, Cambridge, UK; 1:300) overnight at $4^{\circ} \mathrm{C}$. Then, the slides were incubated with rhodamine goat anti-rat antibody (Proteintech, Wuhan, People's Republic of China; 1:200) for $1 \mathrm{~h}$ in the dark. Vascular perfusion was visualized with a fluorescence microscope (Leica DM6000B; Wetzlar, Germany), and the lectin + areas were presented as percentage per CD31+ area.

For vascular permeability, FITC-conjugated dextran $(20 \mathrm{kD})$ was injected $10 \mathrm{~min}$ before tumor capture $(\mathrm{n}=7 /$ group). Tumor tissues were embedded in OCT for cryosection, as described earlier. Leakage quantification was presented as a percentage per CD31+ area.

\section{Hypoxia detection by pimonidazole}

Pimonidazole (Hypoxyprobe, Burlington, MA, USA) was intraperitoneally administered at $60 \mathrm{mg} / \mathrm{kg}, 60 \mathrm{~min}$ before tumors were excised ( $\mathrm{n}=7 /$ group). Samples were fixed with $4 \%$ paraformaldehyde. The detection and location of cells undergoing hypoxia were determined using anti-pimonidazole immunohistochemical staining.

\section{Metastasis assessment}

To assess the formation of distant metastases in mice, we evaluated the incident and number of metastatic nodules in the lungs. After treatment, mice were anesthetized by pentobarbital, and the tumor-bearing footpads were amputated. The lungs were collected 10 days after amputation, fixed with $4 \%$ paraformaldehyde and $6 \mu \mathrm{m}$ thick sections were stained with hematoxylin and eosin (HE) to assess lung metastasis.

\section{Histology and immunostaining}

Tumor-bearing mice were anesthetized with $1 \%$ pentobarbital and perfused with $4 \%$ paraformaldehyde. Tumors were captured and fixed in 4\% paraformaldehyde, and paraffin sections of each group were serially sliced ( $6 \mu \mathrm{m}$ thick) and subjected to immunohistochemical staining using the following antibodies: anti-CD31 antibody (Abcam, 1:500), anti-vimentin antibody (Proteintech, 1:100) and anti-Ecadherin antibody (Abcam, 1:500). The prepared slides were incubated overnight at $4{ }^{\circ} \mathrm{C}$ and then incubated with goat anti-rabbit IgG $(\mathrm{H}+\mathrm{L})$ horseradish peroxidase (Beyotime Institute Biotechnology, Haimen, Jiangsu, People's Republic of China; $1: 100$ ) for $40 \mathrm{~min}$ at $37^{\circ} \mathrm{C}$. The antibody stainings were visualized with 3,3'-diaminobenzidine (DAB). A total of five areas were calculated per sample.

\section{Cell viability assay}

Cells were plated in 96 -well plates $\left(1 \times 10^{4}\right.$ per well $)$ and treated with different agents for $24 \mathrm{~h}$ and $48 \mathrm{~h}$ at $37^{\circ} \mathrm{C}$. Cell viability was assessed by CCK-8 (Dojindo, Kumamoto, Japan). Measurements were carried out at $450 \mathrm{~nm}$ absorbance with an automicroplate reader (Epoch; BioTek, Burlington, VT, USA).

\section{Wound healing assay}

The effects of AuNPs on the migrations of HUVECs and B16F10 cells were evaluated by scratch wound-healing assay. Cells suspended in ECM (HUVECs) and DMEM (B16F10) were seeded on 12-well plates at densities of $2 \times 10^{5} / \mathrm{mL}$. After confirming adhesion to the well-bottoms, the monolayers were scratched manually with sterile $200 \mu \mathrm{L}$ plastic pipette tips and washed thrice with PBS. Cells were allowed to migrate at $37^{\circ} \mathrm{C}, 5 \% \mathrm{CO}_{2}$ for $4 \mathrm{~h}, 8 \mathrm{~h}$ and $12 \mathrm{~h}$. At each time point, images were obtained using an Olympus inverted microscope, and the widths of the wound areas were quantified using ImageJ software (National Institutes of Health).

\section{Western blot}

Total proteins from the cells were extracted with Laemmli buffer, incubated at $90^{\circ} \mathrm{C}$ for $5 \mathrm{~min}$ and centrifuged at $12,000 \times g$ for $20 \mathrm{~min}$. Protein concentrations were measured using the BCA method. For the Western blot assay, proteins were separated on $10 \%$ sodium dodecyl sulfate (SDS) polyacrylamide gel (SDS-PAGE), transferred to polyvinylidene fluoride (PVDF) membranes (Millipore, Bedford, MA, USA), blocked in $5 \%$ bovine serum albumin (BSA) with $0.1 \%$ Tween 20 in tris buffered saline (TBS) for $1 \mathrm{~h}$ at room temperature (RT) and, subsequently, incubated with the following primary antibodies: anti-vimentin antibody (Proteintech, 1:3,000), anti-E-cadherin antibody (Abcam, 1:3,000), anti-ZO-1 antibody (Proteintech, 1:3,000), anti-MMP-2 antibody (Abcam, 1:3,000), anti-c-Myc antibody (Abcam, 1:3,000) and anti-GAPDH antibody (Proteintech, 1:3,000) overnight at $4^{\circ} \mathrm{C}$. Membranes were then incubated with horseradish peroxidase-conjugated secondary antibodies $(1: 2,000$, Thermo Fisher Scientific). The resultant bands were visualized using an enhanced chemiluminescence (ECL) detection system (ChemiDoc XRS; Bio-Rad, Hercules, CA, USA) and quantified using Quantity One software (Bio-Rad).

\section{Statistical analysis}

All data are presented as the mean \pm standard error of mean (SEM). Statistical analysis was carried out using a two-tailed, unpaired Student's $t$-test, and metastasis was assessed using Fisher's exact test. Statistical and graphic analyses were performed 
using GraphPad Prism version 5.0. Significance was attributed for $P<0.05$ ( $* P<0.05$, ** $P<0.01$, and $* * * P<0.001)$.

\section{Results}

\section{Characterization of AuNPs}

The measured UV-vis spectrum displayed a characteristic peak at $\sim 520 \mathrm{~nm}$ (Figure 1A). The hydrodynamic size distribution measured by DLS was 34.09 $\pm 2.4 \mathrm{~nm}$ (Figure 1B), and the FETEM measured actual size was $15 \mathrm{~nm}$ (Figure $1 \mathrm{C}$ and D). The zeta potential was $-31.7 \pm 1.7 \mathrm{mV}$ (Figure 1E), indicating that the nanoparticles were in a relatively stable state in solution.

\section{AuNPs improve vascular morphology}

The endothelial cell marker CD31 was stained to evaluate the impact of AuNPs on tumor angiogenesis. Compared with controls, the average vascular densities after treatment with the AuNPs decreased $(P<0.05$; Figure 2A). Furthermore, the vascular morphology appeared more homogeneous and organized. These results support the hypothesis that AuNPs displayed anti-angiogenesis efficacy.

\section{AuNPs increase vascular perfusion and decrease permeability}

By facilitating tumor vasculature maturation, tumor vessel perfusion could be improved to alleviate hypoxia. ${ }^{17-19}$ Our results from the lectin analysis revealed that tumor blood perfusion significantly increased after AuNPs treatment compared with controls $(P<0.05$; Figure $2 \mathrm{~B})$. The ratio of lectin + to CD31 expression indicated vessels with regular perfusion.

Abnormal tumor blood vessels are often associated with high permeability, which is one of the factors leading to tumor invasion. Our results showed that the leakage of injected dextran into tumor vasculature remarkably decreased after AuNPs treatment $(P<0.05$; Figure 2C). The results
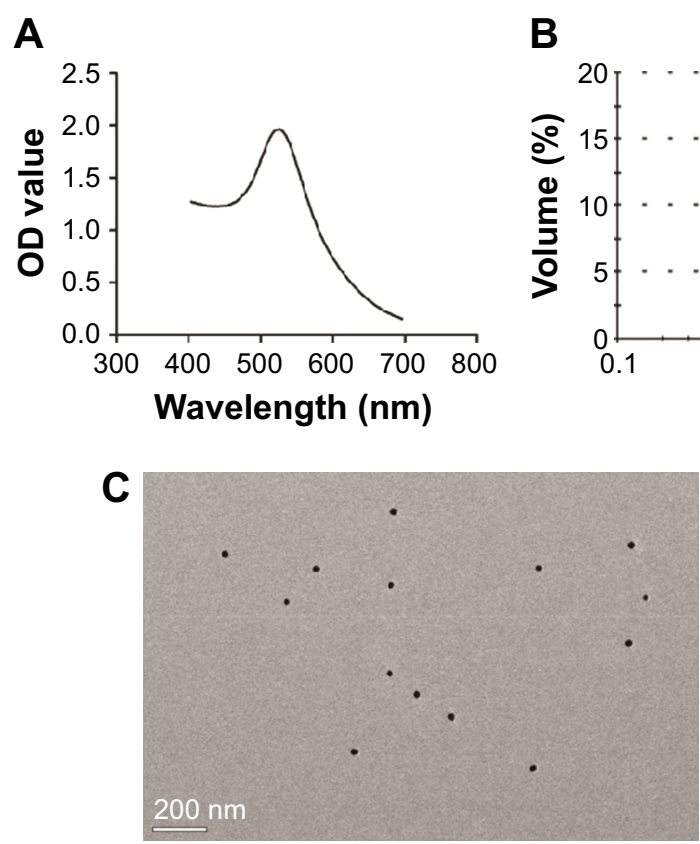
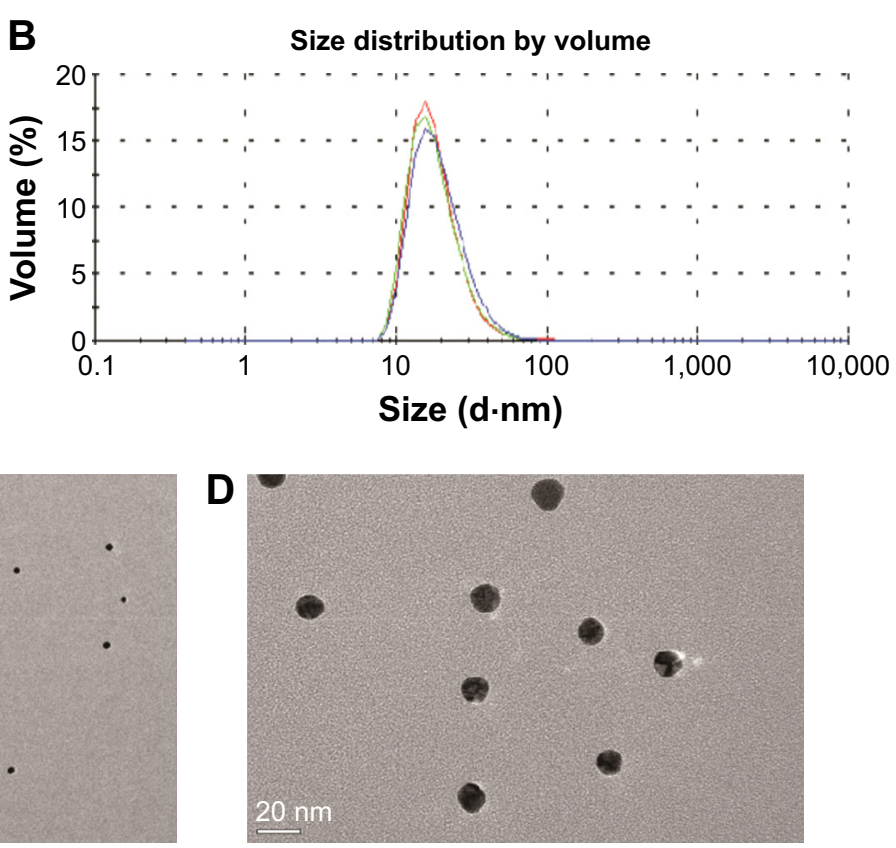

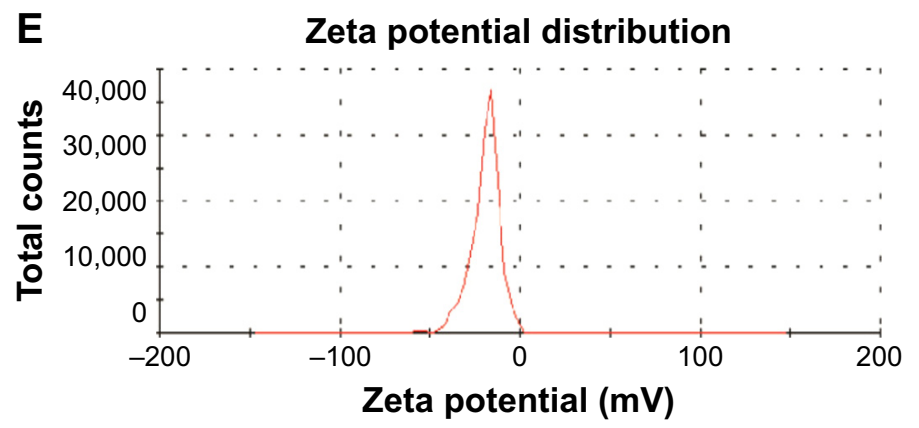

Figure I Characterization of AuNPs.

Notes: (A) UV-visible spectrum showed an absorption peak at $520 \mathrm{~nm}$. (B) The hydrodynamic size distribution was $34.09 \pm 2.4 \mathrm{~nm}$, as measured by DLS. (C) FETEM images of AuNPs (bar: $200 \mathrm{~nm}$ ). (D) FETEM images of AuNPs (bar: $20 \mathrm{~nm}$ ). (E) The zeta potential was $-31.7 \pm 1.7 \mathrm{mV}$, as measured by DLS.

Abbreviations: AuNPs, gold nanoparticles; UV, ultraviolet; DLS, dynamic light scattering; FETEM, field emission transmission electron microscopy; OD, optical density. 
A

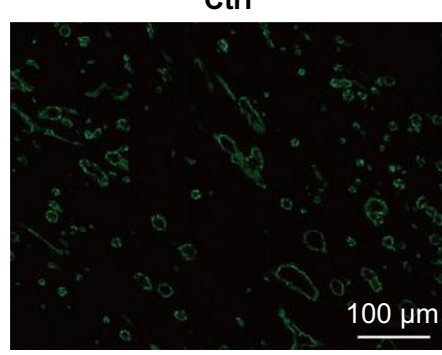

B

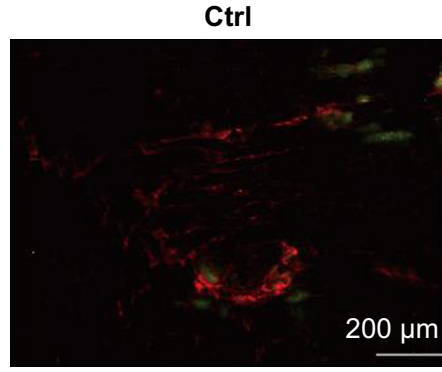

C

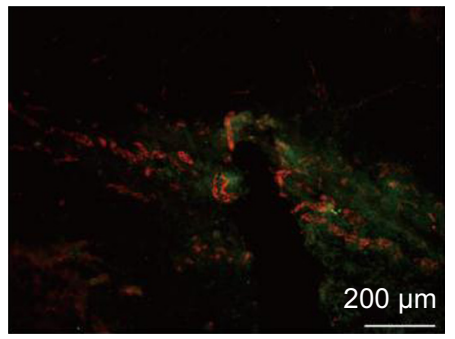

D

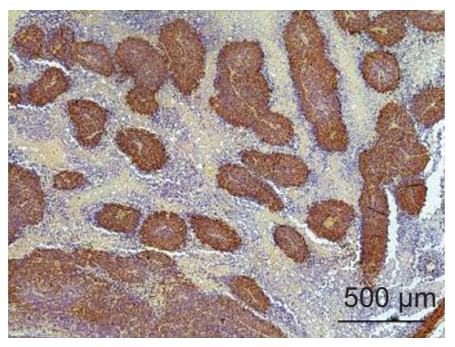

AuNPs

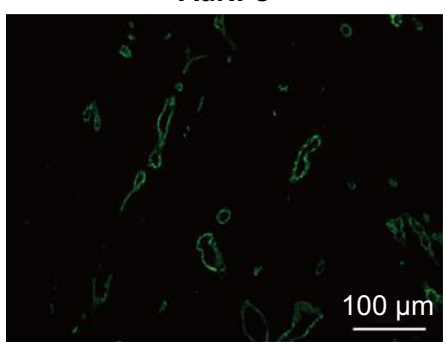

AuNPs
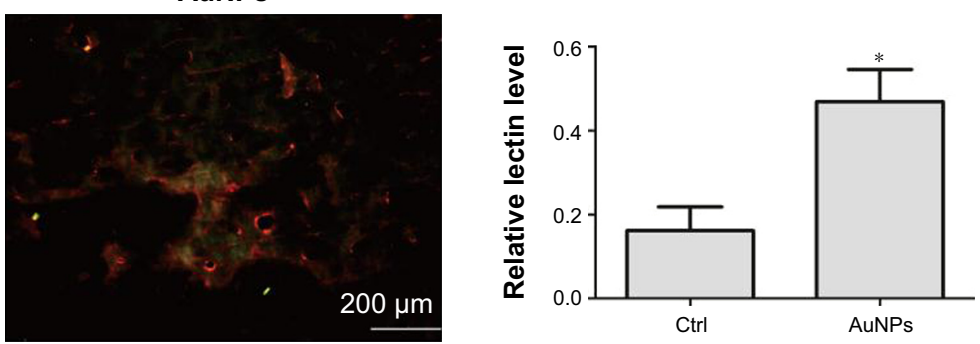

AuNPs
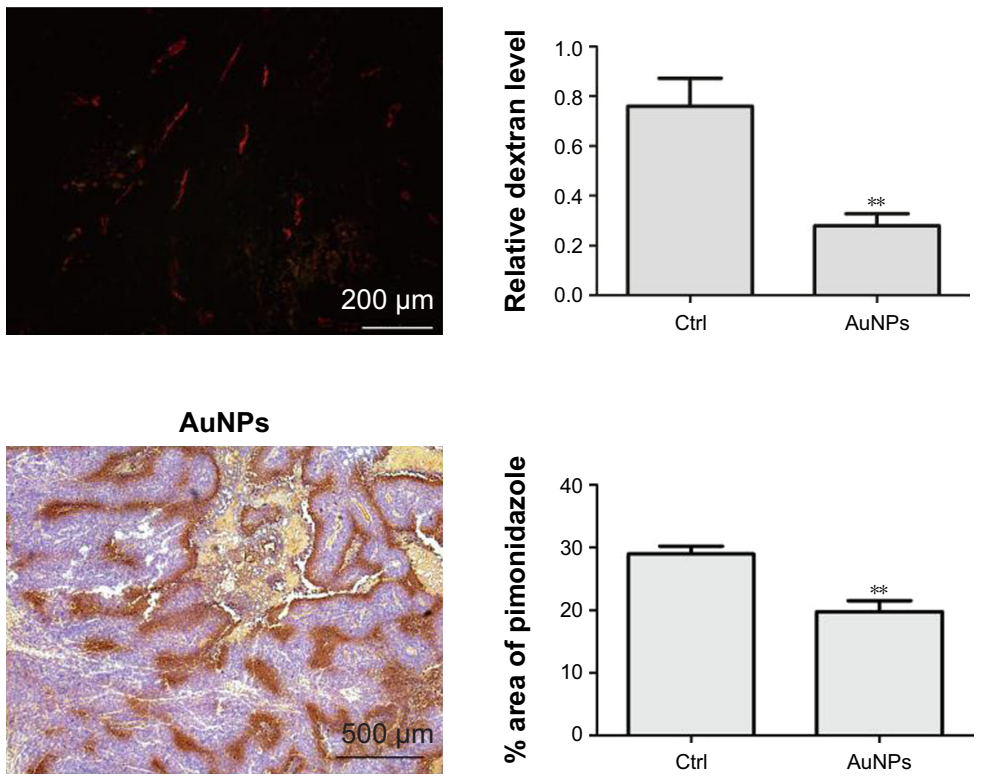

Figure 2 AuNP potential for normalizing tumor vasculature.

Notes: (A) CD3I staining showed the tumor vessel area decreased after treatment with AuNPs. Scale bar $=100 \mu \mathrm{m}$. (B) FITC-conjugated lectin (green) and CD3I (red) staining indicated improved perfusion in tumors after treatment with AuNPs. Scale bar $=200 \mu \mathrm{m}$. (C) FITC-dextran (green) and CD3I staining indicated decreased number of sites of vascular leakage after treatment with AuNPs. Scale bar $=200 \mu \mathrm{m}$. (D) Pimonidazole staining exhibited improvements in hypoxic areas after treatment with AuNPs. Scale bar $=500 \mu \mathrm{m} . * P<0.05$, $* * P<0.01$.

Abbreviations: AuNPs, gold nanoparticles; Ctrl, control; FITC, fluorescein isothiocyanate.

displayed that the maturity of tumor blood vessels was increased by AuNPs treatment.

\section{Alleviation of hypoxia in melanoma tumors}

We next investigated whether the increased blood perfusion was sufficient to decrease hypoxia in tumors.
The immunohistochemical staining for pimonidazole showed that the AuNPs treatment significantly reduced tumor hypoxia $(P<0.05$; Figure $2 \mathrm{D})$, indicating a normal vessel physiology was associated with a reduction in tumor hypoxia. Overall, these results indicated that AuNPs treatment can increase blood perfusion and reduce tumor hypoxia. 


\section{AuNPs reduce the extent of lung metastasis}

Lungs are the most common sites for melanoma metastases. Therefore, we examined the inhibition of metastases in C57BL/6 mice. Three of 15 AuNPs-treated mice and ten of 15 control mice developed lung metastatic nodules at 2 weeks $(P<0.05$; Figure 3A and B). Furthermore, the AuNPs-treated mice exhibited reduced numbers and sizes of lung metastatic colonies (Figure 3C). These results suggested that AuNPsmediated tumor vasculature normalization correlated with reductions in melanoma metastases.

\section{AuNPs inhibit EMT in melanoma tumors}

Vimentin, an important cytoskeletal component, is a marker of mesenchymal cells and a marker of cells undergoing EMT. Our immunohistochemical staining results showed that AuNPs decreased the expression of vimentin in tumors $(P<0.05$; Figure $4 \mathrm{~A}$ and $\mathrm{B})$. Another molecule, E-cadherin, a classical member of the cadherin superfamily, is expressed on cell surfaces in most epithelial tissues. E-cadherin is pivotal for the formation of cell-cell junctions and decreases during the EMT process. In our study, E-cadherin levels increased after AuNPs treatment $(P<0.05$; Figure $4 \mathrm{C}$ and $\mathrm{D})$. The data suggested that treatment using AuNPs reversed the process of EMT in melanoma tumors.

\section{AuNPs have no effects on cell viability but do suppress the migration of HUVECs and BI6FIO cells}

From the wound healing study, we analyzed the migration of B16F10 cells and found that the migration of tumor cells was inhibited $(P<0.05$; Figure $5 \mathrm{~A}$ and $\mathrm{B})$. Additionally, we found that the AuNPs significantly inhibited the migration of HUVECs $(P<0.05$; Figure $5 \mathrm{C}$ and D), which limited the excessive growth of tumor blood vessels and induced the transient normalization of tumor vasculature. On the contrary, data from the CCK- 8 test indicated that the AuNPs had no effect on the cell viability $(P>0.05$; Figure 5E and F). Thus, our data suggested that treatments with AuNPs could inhibit the migration of both HUVECs and tumor cells.

\section{AuNPs inhibit the expression of MMP-2 and c-Myc}

MMP-2 contributes toward tumor invasion and metastases and is involved in tumor angiogenesis. Western blot of tumor protein lysates showed substantially reduced MMP-2 expression levels in the AuNPs-treated group $(P<0.05$; Figure 6A and $\mathrm{B})$. This reduction restricted the invasiveness of tumor cells and directly reduced the probability of metastasis. Similar findings were observed in AuNPs-treated HUVECs $(P<0.05$; Figure 6D and E). Because MMP-2 activity is
A

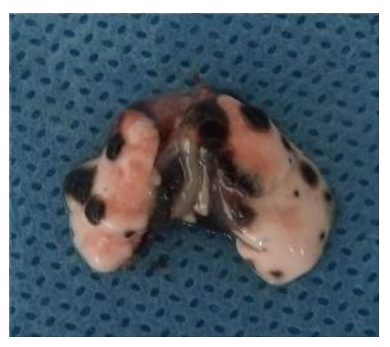

C

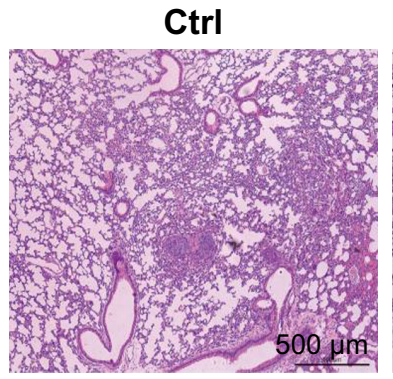

AuNPs

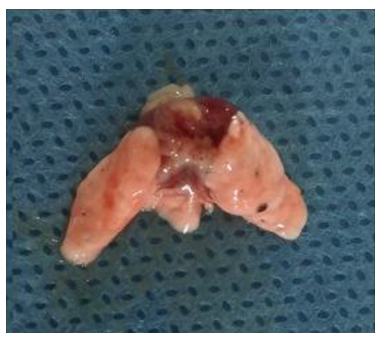

AuNPs

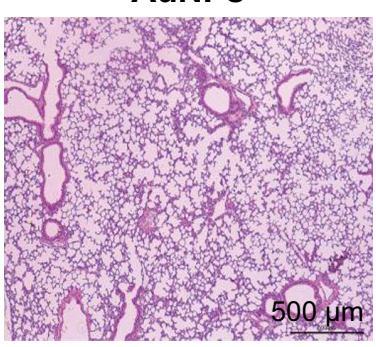

B

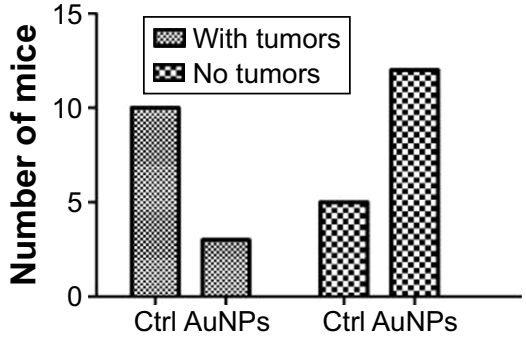

Figure 3 AuNPs reduced the extent of lung metastasis.

Notes: (A) Images of metastatic nodules in lung. (B) Number of mice with metastases. (C) HE staining of metastatic nodules in lung. Scale bar $=500 \mu m$.

Abbreviations: AuNPs, gold nanoparticles; HE, hematoxylin and eosin; Ctrl, control. 
A
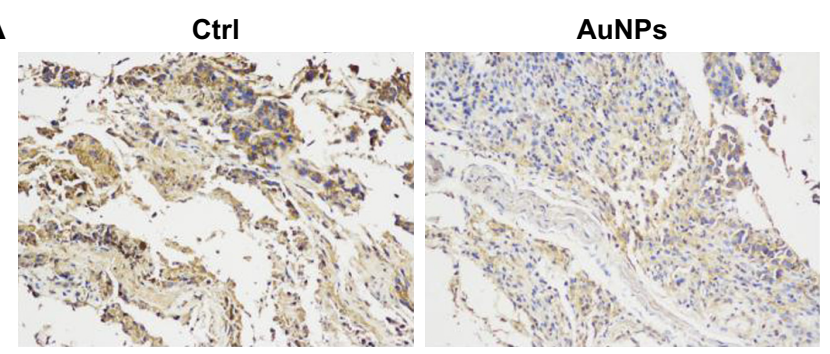

C

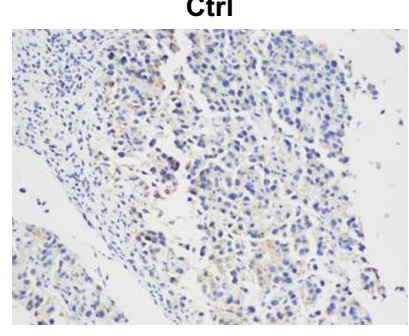

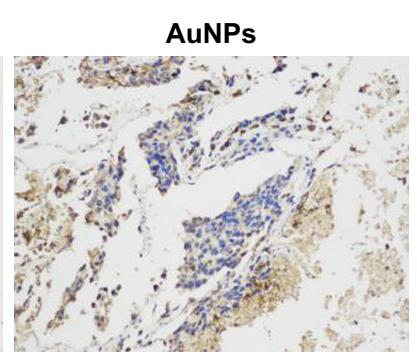

B

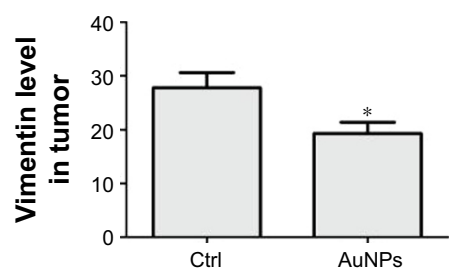

D

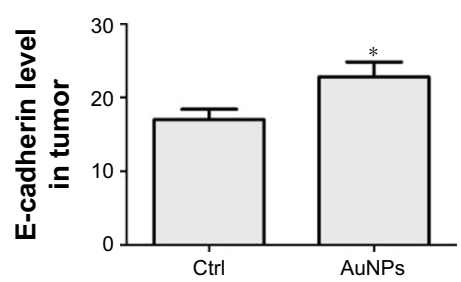

Figure 4 AuNPs inhibit EMT in melanoma tumors.

Notes: (A) Immunohistochemical staining of vimentin in tumor tissues (200x). (B) Quantification of vimentin levels shown in (A). (C) Immunohistochemical staining of E-cadherin expression in tumor tissues (200x). (D) Quantification of E-cadherin levels shown in (C). $* P<0.05$.

Abbreviations: AuNPs, gold nanoparticles; EMT, epithelial-mesenchymal transition; Ctrl, control.

known to drive endothelial cell migration and angiogenesis, ${ }^{20}$ the suppression of MMP-2 reduces the rate of endothelial cell migration and, thereby, inhibits tumor angiogenesis. $\mathrm{ZO}-1$ is a junctional adaptor protein that is essential for endothelial barrier formation; it regulates angiogenesis and is required for normal blood vessel formation. ${ }^{21}$ AuNPs treatment increased the expression levels of ZO-1 $(P<0.05$; Figure $6 \mathrm{D}$ and F) and, thereby, maintained the integrity of endothelial junctions and reduced the probability for tumor cells to escape into circulation. Additionally, c-Myc is a nuclear phosphoprotein that influences cancer progression by promoting EMT or vascularization. In our study, AuNPs treatment decreased c-Myc expression $(P<0.05$; Figure 6A and $\mathrm{C})$. Overall, these data indicated that treatment with AuNPs can inhibit EMT and angiogenesis by regulating MMP-2, c-Myc and ZO-1.

\section{AuNPs reverse vimentin/E-cadherin (V/E) in $\mathrm{BI} 6 \mathrm{FIO}$ cells}

We next investigated whether AuNPs could directly inhibit EMT without improving hypoxia. We analyzed E-cadherin and vimentin levels in B16F10 cells using Western blot. The results revealed that AuNPs increased E-cadherin expression and suppressed mesenchymal phenotype marker vimentin (Figure 6G and H). This indicated that AuNPs directly suppressed EMT in B16F10 cells.

\section{Discussion}

Angiogenesis plays a pivotal role in many physiological and pathological processes, such as organ growth, wound healing and especially tumor progression and metastasis. ${ }^{22-24}$ Rapid expansion renders tumor cells susceptible to hypoxia; as a result, large concentrations of pro-angiogenic factors are released, facilitating excessive angiogenesis in tumors and promoting unstable and heterogeneous morphology and function. Overall, these events lead to increased tumor invasiveness and metastatic potential through a variety of mechanisms.

EMT is a process that induces epithelial cells to lose their adhesiveness and obtain a mesenchymal phenotype. This promotes the detachment of tumor cells from the basement membrane ${ }^{24-26}$ and increases the risk of tumor cells metastasis. Tumor cells undergoing EMT display low cell surface expressions of E-cadherin, which maintain epithelial connections with neighboring cells, yet they further develop a mesenchymal cell-like cytoskeletal structure, including high levels of vimentin, and acquire the ability to leave the primary tumor mass and invade into surrounding tissues and the circulatory system. ${ }^{27}$ There are many factors that contribute to the progression of EMT. One of these factors is hypoxia. ${ }^{28,29}$ Several studies have demonstrated that prolonged anti-angiogenic therapy increased the expression of mesenchymal phenotypes, ${ }^{30-33}$ and this may be due to hypoxia 
A

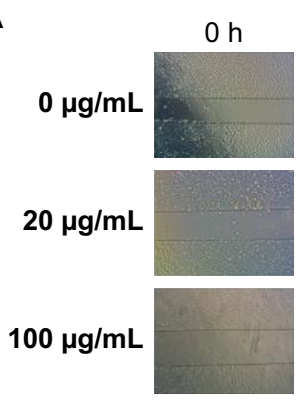

C

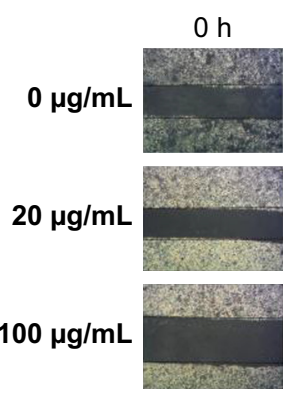

$4 \mathrm{~h}$

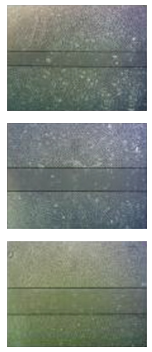

$4 \mathrm{~h}$
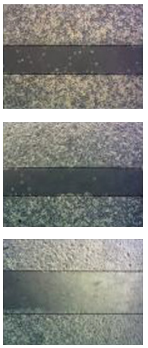

B16F10 $24 \mathrm{~h}$

E

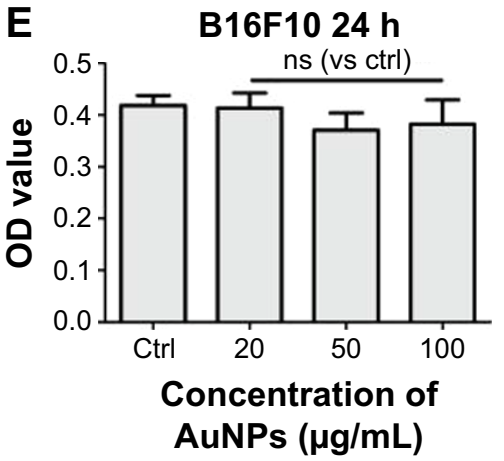

B

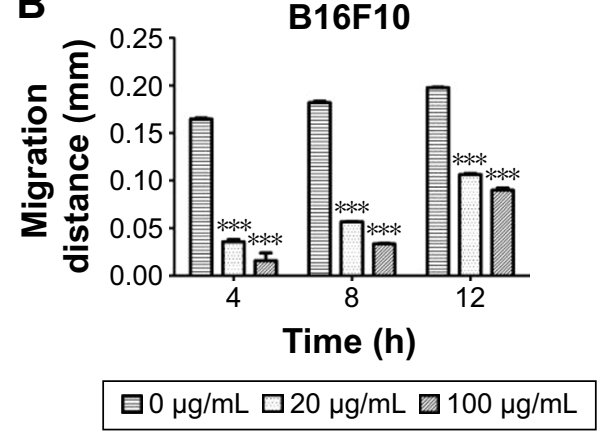

D

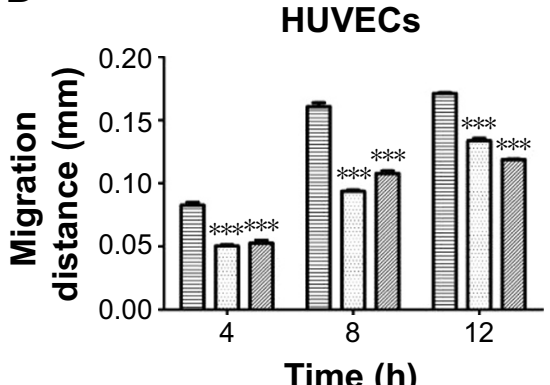

Time (h)

F

HUVECS

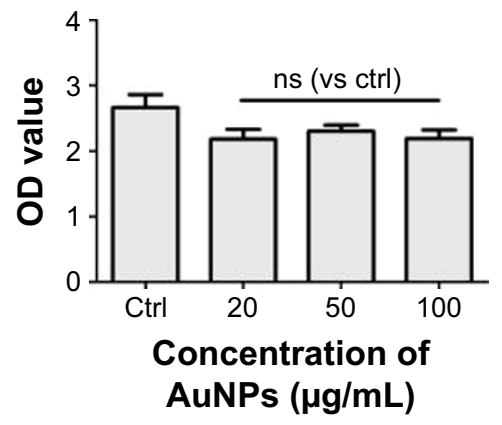

Figure 5 AuNPs suppressed the migration of HUVECs and BI6FI0 cells but had no effects on cell viability.

Notes: (A and B) AuNPs inhibited the migration of BI6FI0 cells at different times. (C and D) AuNPs inhibited the migration of HUVECs at different times. (E and F) AuNPs had no effects on the cell viability of HUVECs and BI6FIO cells. All data are presented as the mean \pm SEM of three independent experiments. ***P $<0.00$ I.

Abbreviations: AuNPs, gold nanoparticles; HUVECs, human umbilical vein endothelial cells; Ctrl, control; OD, optical density; SEM standard error of the mean; ns, no significance; ns, not significant.

induced by excessive anti-angiogenesis. Other studies have also shown that hypoxia and hypoxia-inducible factor-1 alpha $(\mathrm{HIF}-1 \alpha)$ promote EMT in several tumor types. ${ }^{34-36}$

In our study, treatment with AuNPs induced tumor vasculature normalization and decreased hypoxia in tumor sections. These normalized vessels may limit the risk of tumor cells metastasis through blood circulation, and the improvement in oxygenation may subsequently result in a reduction in EMT. Moreover, in B16F10 cells, treatment with AuNPs decreased vimentin and increased E-cadherin levels in the same oxygen environment, resulting in a reversed ratio between the two factors. This indicated that AuNPs could directly inhibit the EMT process independent of improvements in hypoxia. Overall, treatments with AuNPs could directly and indirectly reduce EMT (secondary to improving hypoxic conditions).
A few studies have demonstrated that c-Myc overexpression promotes EMT and facilitates vasculogenic mimicry (VM), ${ }^{37}$ wherein vessel-like structures form via aggressive tumor cells. VM has been linked with prolonged tumor angiogenesis, higher metastatic risk and poor outcomes. Hypoxia conditions induce the formation of VM channels and angiogenesis to acquire a blood supply ${ }^{29,38}$ and subsequently promote EMT. However, EMT also contributes toward VM formation by stimulating tumor cell plasticity and degrading the extracellular matrix (EXCM) to mimic endothelial cells and to form VM channels. ${ }^{29,39}$ In this study, we verified that AuNPs inhibited the expression of c-Myc and regulated the progression of EMT. MMP-2 is involved in the breakdown of EXCM and plays an important role in angiogenesis within tumors, ${ }^{40}$ and hypoxia can activate this 


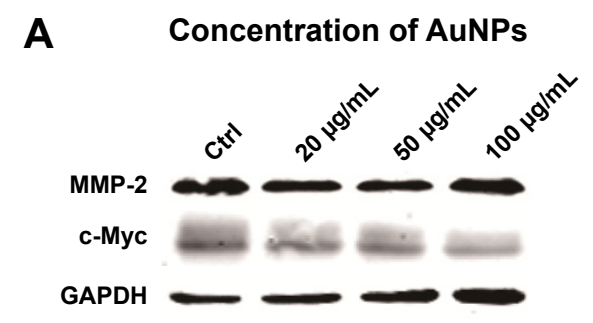

D

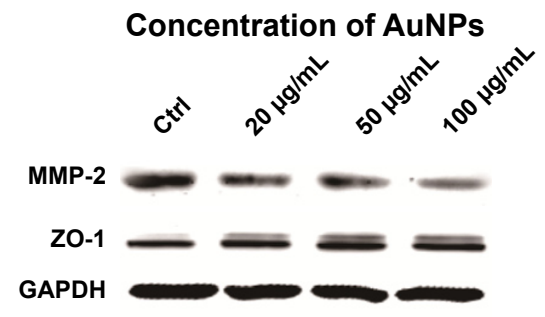

G

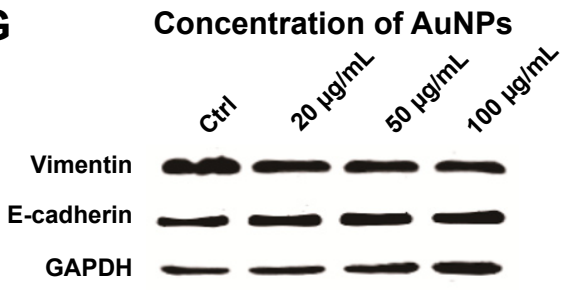

B

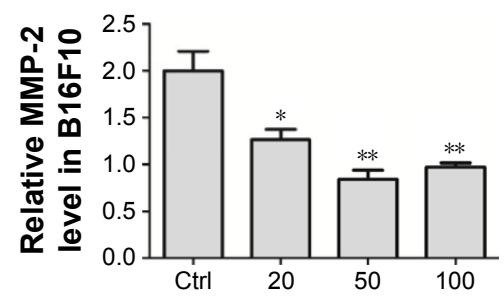

Concentration of AuNPs $(\mu \mathrm{g} / \mathrm{mL})$

E
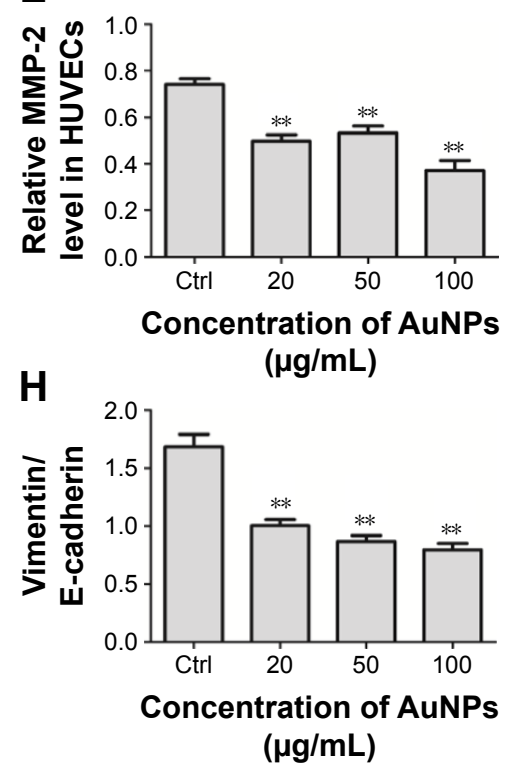

C

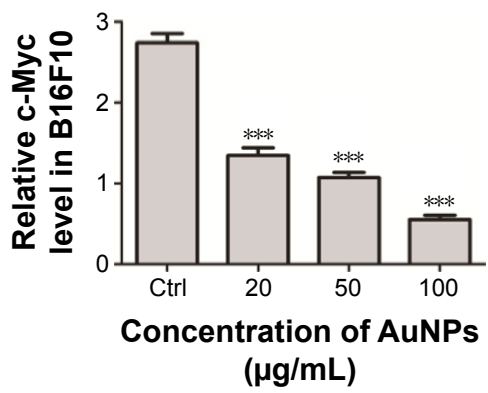

$\mathbf{F}$

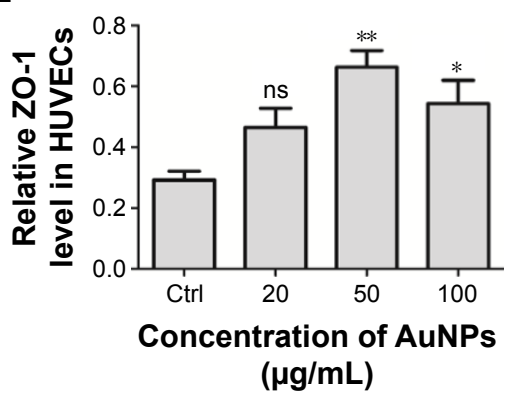

Figure 6 AuNPs inhibited the expression of MMP-2, c-Myc and reversed V/E.

Notes: (A) Western blot analyses for MMP-2 and c-Myc in BI6FI0 cells. (B) AuNPs decreased expression of MMP-2 in BI6FI0 cells. (C) AuNPs decreased expression of c-Myc in BI6FIO cells. (D) Western blot analyses for MMP-2 and ZO-I in HUVECs. (E) AuNPs decreased expression of MMP-2 in HUVECs. (F) AuNPs increased expression of ZO-I in HUVECs. (G) Western blot analyses for V/E in BI6FI0 cells. (H) AuNPs reversed V/E in BI6FI0 cells. *P<0.05, $* * P<0.01$, and $* * * P<0.001$.

Abbreviations: AuNPs, gold nanoparticles; MMP-2, matrix metalloproteinase-2; ZO-I, zonula occludens-I; HUVECs, human umbilical vein endothelial cells; V/E, vimentin/ E-cadherin; Ctrl, control; ns, no significance.

progression to facilitate tumor angiogenesis, invasion and metastasis. Additionally, the overexpression of MMP-2 has been shown to promote EMT. ${ }^{41,42}$ Our data indicated that MMP-2 is another target for AuNPs in the regulation of angiogenesis and EMT.

\section{Conclusion}

We verified that treatment with AuNPs induced tumor vasculature normalization, which decreased hypoxia, strengthened the integrity of the tumor vascular wall and decreased vessel permeability, and our findings provide the first indication that this normalization is associated with the inhibition of MMP2, c-Myc and the promotion of ZO-1. Moreover, further studies proved that AuNPs decreased the expression of VEGF in tumors (Figure S1), which reveals a new mechanism to promote the normalization of tumor blood vessels. Additionally, treatments with AuNPs inhibited EMT in melanoma tumors and in B16F10 cells, and this is a complex process that includes normalization of tumor vessels and the inhibition of MMP-2 and c-Myc. This indicated that AuNPs could directly and indirectly reduce tumor metastasis, and MMP-2 or c-Myc could be a potential therapeutic target. However, more detailed mechanisms should be further explored for clinical benefit.

\section{Acknowledgments}

We thank Dr Xiaoxu Zhao and Hui Ding (Department of General Surgery, The First Affiliated Hospital of Jinan University) for HUVEC isolation and AuNP preparation. This work was supported by the National Natural Science Foundation of China (81472849), the Guangdong Natural Science Research (2014A030313383) and the Guangdong High-level University Construction Fund for Jinan University (88016013034). 


\section{Disclosure}

The authors report no conflicts of interest in this work.

\section{References}

1. Wong MLH, Prawira A, Kaye AH, Hovens CM. Tumour angiogenesis: its mechanism and therapeutic implications in malignant gliomas. J Clin Neurosci. 2009;16(9):1119-1130.

2. Goel S, Wong AHK, Jain RK. Vascular normalization as a therapeutic strategy for malignant and nonmalignant disease. Cold Spring Harb Perspect Med. 2012;2(3):a006486.

3. DeClerck K, Elble RC. The role of hypoxia and acidosis in promoting metastasis and resistance to chemotherapy. Front Biosci. 2010;15: 213-225.

4. Jain RK, Duda DG, Clark JW, Loeffler JS. Lessons from phase III clinical trials on anti-VEGF therapy for cancer. Nat Clin Pract Oncol. 2006;3(1):24-40.

5. McIntyre A, Harris AL. Metabolic and hypoxic adaptation to antiangiogenic therapy: a target for induced essentiality. EMBO Mol Med. 2015;7(4):368-379.

6. Stathopoulos GP, Batziou C, Trafalis D, et al. Treatment of colorectal cancer with and without bevacizumab: a phase III study. Oncology. 2010;78(5-6):376-381.

7. Koeberle D, Betticher DC, von Moos R, et al. Bevacizumab continuation versus no continuation after first-line chemotherapy plus bevacizumab in patients with metastatic colorectal cancer: a randomized phase III non-inferiority trial (SAKK 41/06). Ann Oncol. 2015;26(4): 709-714.

8. de Gramont A, Van Cutsem E, Schmoll HJ, et al. Bevacizumab plus oxaliplatin-based chemotherapy as adjuvant treatment for colon cancer (AVANT): a phase 3 randomised controlled trial. Lancet Oncol. 2012; 13(12):1225-1233.

9. Paez-Ribes M, Allen E, Hudock J, et al. Antiangiogenic therapy elicits malignant progression of tumors to increased local invasion and distant metastasis. Cancer Cell. 2009;15(3):220-231.

10. Jain RK. Normalizing tumor vasculature with anti-angiogenic therapy: a new paradigm for combination therapy. Nat Med. 2001;7(9): 987-989.

11. Maeda H, Nakamura H, Fang J. The EPR effect for macromolecular drug delivery to solid tumors: improvement of tumor uptake, lowering of systemic toxicity, and distinct tumor imaging in vivo. Adv Drug Deliv Rev. 2013;65(1):71-79.

12. Li Y, Lian Y, Zhang LT, et al. Cell and nanoparticle transport in tumour microvasculature: the role of size, shape and surface functionality of nanoparticles. Interface Focus. 2016;6(1):20150086.

13. Li W, Zhao X, Du B, et al. Gold nanoparticle-mediated targeted delivery of recombinant human endostatin normalizes tumour vasculature and improves cancer therapy. Sci Rep. 2016;6:30619.

14. Pan Y, Ding H, Qin L, Zhao X, Cai J, Du B. Gold nanoparticles induce nanostructural reorganization of VEGFR2 to repress angiogenesis. J Biomed Nanotechnol. 2013;9(10):1746-1756.

15. Pan Y, Wu Q, Liu R, et al. Inhibition effects of gold nanoparticles on proliferation and migration in hepatic carcinoma-conditioned HUVECs. Bioorg Med Chem Lett. 2014;24(2):679-684.

16. Pan YL, Wu Q, Qin L, Cai JY, Du B. Gold nanoparticles inhibit VEGF(165)-induced migration and tube formation of endothelial cells via the Akt pathway. Biomed Res Int. 2014;2014:418624.

17. Leuci V, Maione F, Rotolo R, et al. Lenalidomide normalizes tumor vessels in colorectal cancer improving chemotherapy activity. J Transl Med. 2016;14(1):119.

18. Peterson TE, Kirkpatrick ND, Huang YH, et al. Dual inhibition of Ang-2 and VEGF receptors normalizes tumor vasculature and prolongs survival in glioblastoma by altering macrophages. Proc Natl Acad Sci US A. 2016;113(16):4470-4475.
19. Batchelor TT, Gerstner ER, Emblem KE, et al. Improved tumor oxygenation and survival in glioblastoma patients who show increased blood perfusion after cediranib and chemoradiation. Proc Natl Acad Sci U S A. 2013;110(47):19059-19064.

20. Ma YL, Lin SW, Fang HC, et al. A novel poly-naphthol compound ST104P suppresses angiogenesis by attenuating matrix metalloproteinase-2 expression in endothelial cells. Int J Mol Sci. 2014;15(9):16611-16627.

21. Tornavaca O, Chia M, Dufton N, et al. ZO-1 controls endothelial adherens junctions, cell-cell tension, angiogenesis, and barrier formation. J Cell Biol. 2015;208(6):821-838.

22. Carmeliet P. Angiogenesis in life, disease and medicine. Nature. 2005; 438(7070):932-936.

23. Folkman J. Angiogenesis in cancer, vascular, rheumatoid and other disease. Nat Med. 1995;1(1):27-31.

24. Carmeliet P, Jain RK. Angiogenesis in cancer and other diseases. Nature. 2000;407(6801):249-257.

25. Iwadate Y. Epithelial-mesenchymal transition in glioblastoma progression. Oncol Lett. 2016;11(3):1615-1620.

26. Cichon MA, Radisky DC. ROS-induced epithelial-mesenchymal transition in mammary epithelial cells is mediated by NF-kappa B-dependent activation of Snail. Oncotarget. 2014;5(9):2827-2838.

27. Patel A, Sabbineni H, Clarke A, Somanath PR. Novel roles of Src in cancer cell epithelial-to-mesenchymal transition, vascular permeability, microinvasion and metastasis. Life Sci. 2016;157:52-61.

28. Wong PP, Demircioglu F, Ghazaly E, et al. Dual-action combination therapy enhances angiogenesis while reducing tumor growth and spread. Cancer Cell. 2015;27(1):123-137.

29. Liu K, Sun B, Zhao X, et al. Hypoxia induced epithelial-mesenchymal transition and vasculogenic mimicry formation by promoting Bcl-2/ Twist1 cooperation. Exp Mol Pathol. 2015;99(2):383-391.

30. Xu H, Rahimpour S, Nesvick CL, et al. Activation of hypoxia signaling induces phenotypic transformation of glioma cells: implications for bevacizumab antiangiogenic therapy. Oncotarget. 2015;6(14): 11882-11893.

31. Piao Y, Liang J, Holmes L, Henry V, Sulman E, de Groot JF. Acquired resistance to anti-VEGF therapy in glioblastoma is associated with a mesenchymal transition. Clin Cancer Res. 2013;19(16):4392-4403.

32. Carbone C, Moccia T, Zhu C, et al. Anti-VEGF treatment-resistant pancreatic cancers secrete proinflammatory factors that contribute to malignant progression by inducing an EMT cell phenotype. Clin Cancer Res. 2011;17(17):5822-5832.

33. Hammers HJ, Verheul HM, Salumbides B, et al. Reversible epithelial to mesenchymal transition and acquired resistance to sunitinib in patients with renal cell carcinoma: evidence from a xenograft study. Mol Cancer Ther. 2010;9(6):1525-1535.

34. Zhang WJ, Shi XP, Peng Y, et al. HIF-1 alpha promotes epithelialmesenchymal transition and metastasis through direct regulation of ZEB1 in colorectal cancer. PLoS One. 2015;10(6): e0129603.

35. Li M, Wang YX, Luo Y, et al. Hypoxia inducible factor-1alphadependent epithelial to mesenchymal transition under hypoxic conditions in prostate cancer cells. Oncol Rep. 2016;36(1):521-527.

36. Li S, Zhang J, Yang H, Wu CH, Dang XT, Liu YY. Copper depletion inhibits $\mathrm{CoCl} 2$-induced aggressive phenotype of MCF-7 cells via downregulation of HIF-1 and inhibition of Snail/Twist-mediated epithelial-mesenchymal transition. Sci Rep. 2015;5:12410.

37. Lin X, Sun R, Zhao X, et al. C-myc overexpression drives melanoma metastasis by promoting vasculogenic mimicry via c-myc/snail/Bax signaling. J Mol Med. 2017;95(1):53-67.

38. Sun BC, Zhang DF, Zhang SW, Zhang WZ, Guo H, Zhao XL. Hypoxia influences vasculogenic mimicry channel formation and tumor invasionrelated protein expression in melanoma. Cancer Lett. 2007;249(2): $188-197$.

39. Sun B, Zhang D, Zhao N, Zhao X. Epithelial-to-endothelial transition and cancer stem cells: two cornerstones of vasculogenic mimicry in malignant tumors. Oncotarget. Epub 2016 Mar 29. 
40. Gao F, Sun M, Gong Y, Wang H, Wang Y, Hou H. MicroRNA-195a-3p inhibits angiogenesis by targeting Mmp2 in murine mesenchymal stem cells. Mol Reprod Dev. 2016;83(5):413-423.

41. Gialeli C, Theocharis AD, Karamanos NK. Roles of matrix metalloproteinases in cancer progression and their pharmacological targeting. FEBS J. 2011;278(1):16-27.
42. Bae GY, Choi SJ, Lee JS, et al. Loss of E-cadherin activates EGFR-MEK ERK signaling, which promotes invasion via the ZEB1/MMP2 axis in non-small cell lung cancer. Oncotarget. 2013;4(12):2512-2522. 


\section{Supplementary materials}

In this study, we did immunohistochemical analysis to explore what happened to VEGF after treated by gold nanoparticles (AuNPs). Our data showed that AuNPs decreased VEGF expression in melanoma tumors $(P<0.05$; Figure S1A-C). However, no effects on VEGFR2 expression were observed after AuNP treatment (Figure S1D-F). Thus, we thought the AuNPs may inhibit the VEGF-VEGFR2 pathway by the following mechanisms: 1) decrease the expression of VEGF in tumors; 2) reduce VEGF165-induced VEGFR2 phosphorylation; 3) inhibit VEGF165-VEGFR2 interaction; 4) suppress the formation of nanodomains of VEGFR2.
A

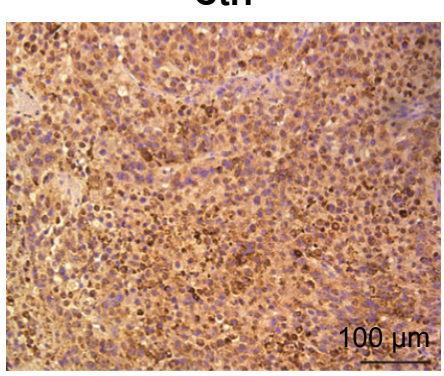

D

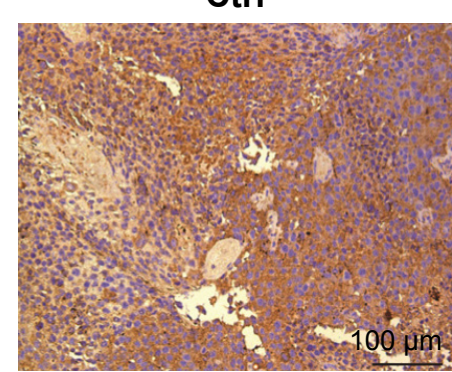

B

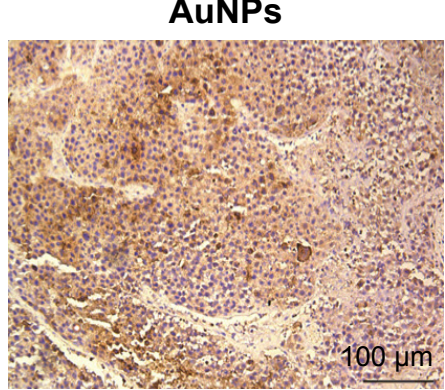

E

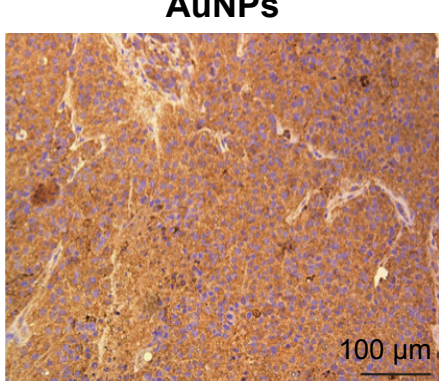

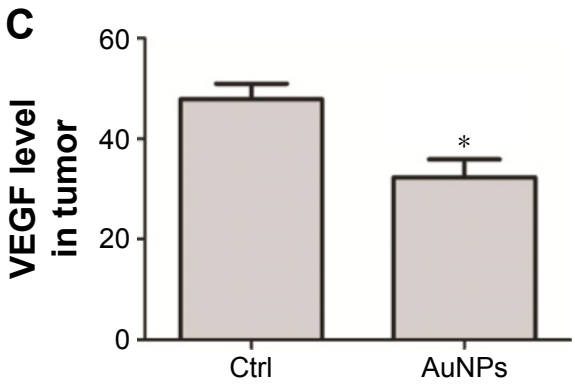

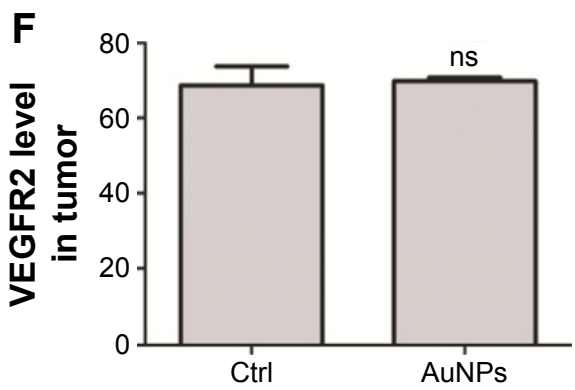

Figure SI Effects of AuNPs on VEGF and VEGFR2 in melanoma tumor.

Notes: (A and B) Immunohistochemical staining of VEGF in tumor tissues (bars: $100 \mu \mathrm{m}$ ). (C) Quantification of VEGF levels shown in (A and B). (D and E) Immunohistochemical staining of VEGFR2 expression in tumor tissues (bars: $100 \mu \mathrm{m})$. (F) Quantification of VEGFR2 levels shown in (D and $\mathbf{E}) . * P<0.05$.

Abbreviations: AuNPs, gold nanoparticles; Ctrl, control; ns, no significance.

\section{Publish your work in this journal}

The International Journal of Nanomedicine is an international, peerreviewed journal focusing on the application of nanotechnology in diagnostics, therapeutics, and drug delivery systems throughout the biomedical field. This journal is indexed on PubMed Central, MedLine, CAS, SciSearch $®$, Current Contents ${ }^{\circledR} /$ Clinical Medicine,
Journal Citation Reports/Science Edition, EMBase, Scopus and the Elsevier Bibliographic databases. The manuscript management system is completely online and includes a very quick and fair peer-review system, which is all easy to use. Visit http://www.dovepress.com/ testimonials.php to read real quotes from published authors. 